
allemande

51-2| 2019

Les Humanités environnementales : circulations et renouvellement des savoirs en France et en Allemagne

\title{
Ecocriticism/écocritique im deutschen und französischen Kontext: eine vergleichende Perspektive
}

\section{Aurélie Choné}

\section{(Q) OpenEdition}

\section{Journals}

Édition électronique

URL : https://journals.openedition.org/allemagne/1975

DOI : 10.4000/allemagne. 1975

ISSN : 2605-7913

Éditeur

Société d'études allemandes

Édition imprimée

Date de publication : 10 décembre 2019

Pagination : 321-341

ISSN : 0035-0974

Référence électronique

Aurélie Choné, „Ecocriticism/écocritique im deutschen und französischen Kontext: eine vergleichende Perspektive", Revue d'Allemagne et des pays de langue allemande [Online], 51-2 | 2019, Online erschienen am: 02 Dezember 2020, abgerufen am 20 Mai 2021. URL: http://journals.openedition.org/allemagne/ 1975 ; DOI: https://doi.org/10.4000/allemagne. 1975 


\title{
Ecocriticism/écocritique im deutschen und französischen Kontext: eine vergleichende Perspektive
}

\author{
- Aurélie Choné *
}

\section{Einleitung}

Angesichts der verschiedenen kulturellen Traditionen und Umweltdiskursen in Deutschland und Frankreich ist eine komparatistische Perspektive auf die neuen Paradigmen einer umweltbezogenen Literaturwissenschaft sicher aufschlussreich, um sowohl Begriffe wie Natur, Landschaft, Garten, Wald, Wildnis (sowie ihre konkreten Umsetzungen), als auch den Umgang mit der Natur/Kultur-Dichotomie und mit den Environmental Humanities in beiden kulturellen Kontexten besser zu verstehen. Ein positives Ergebnis dieser Studie wäre es, eine gemeinsame Grundlage ans Licht zu bringen, die nicht nur zum besseren gegenseitigen Verständnis, sondern auch zu künftiger deutsch-französischer Zusammenarbeit verhelfen könnte. Mein Anliegen ist, den deutschen Ecocriticism bzw. die französische écocritique in kulturwissenschaftlichen Traditionen und unter Berücksichtigung der Eigenheiten der politischen und gesellschaftlichen Auseinandersetzung mit Natur und Umwelt im deutschen bzw. französischen Kontext zu situieren, um Wege aufzuzeigen, wie sich eine deutsch-französische Komparatistik im Feld der Forschungen zum Thema „Literatur und Ökologie“ besser entwickeln kann.

Folgende Fragestellungen wären für eine deutsch-französische Untersuchung von ökologisch orientierten Literatur- und Kulturwissenschaften relevant: Welche Unterschiede zwischen den Konzepten und Praktiken von Ecocriticism ${ }^{(1)}$ fallen auf? Lassen sich spezifische Ansätze des Ecocriticism im französischen und im deutschen Kontext identifizieren? Welche Beziehungen unterhalten sie zu Feldern wie Cultural Studies, Sozialwissenschaften, Environmental Humanities und Medienwissenschaften sowie zu nahe liegenden Ansätzen wie New Materialism, Postcolonial Studies, Feminist

* Dozentin für Neuere Deutsche Literatur und Ideengeschichte am Institut für Germanistik der Universität Straßburg, EA 1341 Mondes germaniques et nord-européens, achone@unistra.fr.

1 Aus praktischen Gründen verwende ich in diesem Absatz den Begriff Ecocriticism für beide Kontexte. 
Studies oder Ökopoetik? Erklären sich die Unterschiede durch verschiedene kulturelle Traditionen der Naturforschung? Auf welche Referenztexte nehmen die französischen bzw. deutschen Wissenschaftlerinnen und Wissenschaftler Bezug? Wie inter- und transdisziplinär sind ihre Praktiken des Ecocriticism? Im vorliegenden Beitrag können natürlich nur einige Fragen genauer untersucht werden. Vor allem wird erforscht, wie sich die beiden Felder in Deutschland und Frankreich entwickelt haben und welche Schnittpunkte/Gemeinsamkeiten sich ergeben. Ohne Anspruch auf Vollständigkeit werden die jeweiligen Entwicklungslinien nachgezeichnet.

Interessant ist zuerst zu bemerken, dass der Begriff Ecocriticism in beiden kulturellen Kontexten andere epistemologische Fragestellungen aufwirft und andere Arbeitsmethoden voraussetzt. Im deutschen Kontext konnte der Begriff Ökokritik sich offensichtlich nicht durchsetzen, obwohl er in Frankreich als écocritique seit den 2000er Jahren fest etabliert ist. Wahrscheinlich klingt das „Öko“-Präfix zu politisch, propagandistisch und klischeehaft und die „Kritik“ würde auf etwas anderes als eine philologische, literatur- und kulturwissenschaftliche Herangehensweise verweisen. Wenn in Deutschland vor allem der Begriff Ecocriticism Verwendung findet, liegt das zum Teil daran, dass er nicht so lang und umständlich ist wie der Ausdruck „umweltbezogene Literatur- und Kulturwissenschaften“, zum anderen knüpft er an eine erweiterte Auffassung des „criticism“ an, die sich im Zuge der Rezeption des (Post)strukturalismus in den USA zusammen mit anderen Ansätzen wie der French Theory (Foucault, Derrida, usw.) etablierte ${ }^{(2)}$. Allerdings ist der Begriff Ecocriticism im deutschen Kontext noch nicht ganz stabilisiert, wie es andere konkurrierende Termini bezeugen, zum Beispiel das Feld „Literatur und Ökologie“(3), „Literatur- und Umwelt-Studien“(4) (analog zu „Literature and the Environment") oder „Natureculture Studies“, in Bezug auf Donna Haraway ${ }^{(5)}$.

\section{US-amerikanischer versus europäischer Ecocriticism: die Entstehung eines Forschungsfeldes}

Obwohl sich die Umweltbewegung schon in den 1960er Jahren verbreitete, entstand der Ansatz des Ecocriticism erst Anfang der 1990er Jahre aus dem Umfeld der

2 Timo Müller, „Kritische Theorie und Ecocriticism“, in: Gabriele Dürbeck und Urte Stobbe (Hg.), Ecocriticism: Eine Einführung, Köln, Böhlau, 2015, S. 160-171; Andrew Arato, „Die Kritische Theorie in den USA. Gedanken über vier Jahrzehnte ihrer Rezeption“, in: Frank Trommler (Hg.), Amerika und die Deutschen, Wiesbaden, Springer Fachmedien, 1986, S. 227-234.

3 So erstmals Axel Goodbody (Hg.), Literatur und Ökologie, Amsterdam, Rodopi (Amsterdamer Beiträge Zur Neueren Germanistik, Bd. 43), 1998. Und wieder: Claudia Schmit t und Christiane SolteGresser (Hg.) Literatur und Ökologie. Neue literatur- und kulturwissenschaftliche Perspektiven, Bielefeld, Aisthesis Verlag, 2017.

4 Ich verweise hier auf die von Gabriele Dürbeck, Hannes Bergthaller, Robert S. Emmett, Serenella Iovino und Ulrike Plath herausgegebene Buchreihe „Studien zu Literatur, Kultur und Umwelt/ Studies in Literature, Culture, and the Environment" beim Verlag Peter Lang.

5 Interessant in der Perspektive einer Zirkulation von Wissen ist zu beobachten, dass der französische Soziologe und Philosoph Bruno Latour auf die US-amerikanische Naturwissenschaftsphilosophin und Frauenforscherin Donna Haraway und auf den sogenannten material turn Einfluss hatte, d.h. auf die Neubewertung der Materie, des Körpers, die wiederum relativ mehr Resonanz in Deutschland als in Frankreich fand. Siehe Tobias S. Goll, Daniel KeIl und Thomas Telios (Hg.), Critical Matter. Diskussionen eines neuen Materialismus, Münster, edition assemblage (Reihe kritik_praxis, Bd. 2), 2013. 
angelsächsischen Literatur- und Kulturwissenschaften. 1993 wurde die Association for the Study for Literature and Environment (ASLE) in Nevada gegründet. Ecocriticism hat sich als neues interdisziplinäres Forschungsgebiet herausgebildet, um das Verhältnis von Literatur und Umwelt, unter anderem durch Einbeziehung ökologischer Aspekte in literarischen Texten zu erforschen. Erstmals erschienen Pionierwerke in den USA, wie Joseph Meekers The Comedy of Survival (1974) und William Rueckerts Essay Literature and Ecology: An Experiment in Ecocriticism (1978) - wo der Begriff „Ecocriticism“ zum ersten Mal auftaucht. Später erschienen zwei bahnbrechende Werke: Laurence Buells The Environmental Imagination ${ }^{(6)}$ und die von Cheryll Glotfelty und Harold Fromm herausgegebene Anthologie The Ecocriticism Reader: Landmarks in Literary Ecology. Cheryll Glotfelty zufolge sei Ecocriticism (Abkürzung von „ecological criticism") ,the study of the relationship between literature and the phy-

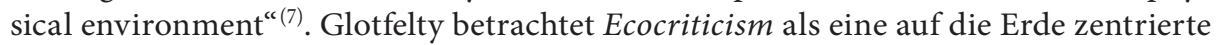
(„earth-centered“) Herangehensweise der literarischen Studien ${ }^{(8)} .1991$ wurde die USamerikanische Association for the Study of Literature and Environment gegründet und 1993 die Zeitschrift Interdisciplinary Studies in Literature and Environment. In Europa etablierte sich dieser methodologische Ansatz erst am Anfang des 21. Jahrhunderts mit der Gründung der European Association for the Study of Literature, Culture, and Environment im Jahr 2004 und der Zeitschrift Ecozon@ 2010. Dennoch erfreuen sich Forschungen zum Ecocriticism bis heute nicht der gleichen Sichtbarkeit und Anerkennung in Europa wie in den USA.

Angesichts dieser zeitlichen Verschiebung könnte die Frage aufgeworfen werden, weshalb der Ecocriticism in Europa später Fuß fasste und auf geringeres Interesse stieß. Eine Erklärung könnte vielleicht in der europäischen Mentalitätsgeschichte und im Trauma des 2. Weltkriegs gefunden werden. Seitdem Tier- und Umweltschutz zur Ideologie der Nazis wurde ${ }^{(9)}$, gilt die Beschäftigung mit der Natur in Europa, insbesondere in Deutschland und in Frankreich, als eher konservativ und erweckt schnell den Verdacht, sich nicht genügend von einer nazistisch geprägten „Blut-und-Boden“Ideologie zu distanzieren. In Deutschland konnte z.B. der wichtige ökokritische Begriff „Sense of place“(10) (manchmal als „Heimatbewusstsein“ übersetzt) aufgrund seiner Konnotation mit „,ölkischem Denken“, die auf die Verbindung von Natur und nationaler Identität hindeutet, nur schwer Fuß fassen.

Dazu kommt, dass die im US-amerikanischen Ecocriticism behandelten Themen - wie der Wilderness-Begriff und der Frontier-Mythos - der europäischen Tradition vermutlich eher fremd sind. Während in der (US-)amerikanischen Ausprägung des Ecocriticism das literarische Genre nature writing von großer Bedeutung ist, sind in der europäischen Tradition andere Gattungen wie die Pastorale, die Idylle und die

6 Lawrence BuelL, The Environmental Imagination, London, Harvard UP, 1995.

7 Cheryll Glotfelty und Harold Fromm (Hg.), The Ecocriticism Reader: Landmarks in Literary Ecology, University of Georgia Press, 1996, Introduction, S. xviii.

8 Ebd.: „Ecocriticism takes an earth-centered approach to literary studies“.

9 Peter Bierl, Grüne Braune. Umwelt-, Tier- und Heimatschutz von rechts, Münster, Unrast Verlag, 2014.

10 Ursula K. Heise, Sense of Place and Sense of Planet: The Environmental Imagination of the Global, Oxford, Oxford University Press, 2008. 
Bukolik verankert. Die Begegnung mit einer unberührten Wildnis (Wilderness) ist für den nordamerikanischen Kontext typisch und gehört nicht zur europäischen Tradition, wo die Natur nicht als eine Welt abseits der Menschheit, sondern immer als sozial und untrennbar von der menschlichen Geschichte betrachtet wird, was besonders für das Agrarland Frankreich und das Waldland Deutschland zutrifft. Als wichtige kulturelle Unterschiede nennt der Literaturwissenschaftler Bergthaller noch die besonders protestantische Färbung ökologischen Denkens in den USA und die wesentliche Rolle von Ansätzen der deep ecolog $y^{(11)}$, die lange in Europa des Ökofaschismus ${ }^{(12)}$ und eines naiv-romantischen Naturbildes ${ }^{(13)}$ bezichtigt wurden.

Ein anderer Grund könnte sein, dass die Traditionen der Naturforschung, die in Europa, insbesondere in Deutschland und Frankreich stark vertreten sind, eher durch das Medium der Sachbücher vermittelt wurden, was zu ihrer Verbreitung wenig bei$\operatorname{trug}^{(14)}$. Dazu kommt, dass die größten Romane der deutschen und französischen Literatur Umweltthemen nicht besonders in den Vordergrund gestellt haben, im Gegensatz zu Werken von Thoreau, Muir, Hardy usw. Als Hemmungsfaktor für einen einheitlichen europäischen Ecocriticism nennen Rigby und Goodbody auch die Vielsprachigkeit des europäischen Kontinents mit seinen unterschiedlichen Traditionen ${ }^{(15)}$. Als weiteres Hemmnis für die Entwicklung einer europäischen Ökokritik erwähnen Schmitt und Solte-Gresser schließlich „die traditionelle Skepsis gegenüber politisch engagierten Forschungsansätzen, und zum anderen die Tatsache, dass ökologisches Denken in Europa, trotz aller Kritik am Anthropozentrismus, zumeist einer gewissen Fortschritts- und Anpassungslogik folgt “(16). Aus diesen Gründen erklärt sich die Dominanz US-amerikanischer Literatur in europäischen ökokritischen Studien bis heute. Der deutsche Ecocriticism ${ }^{(17)}$ und die französische écocritique ${ }^{(18)}$ wurden zuerst von Amerikanisten eingeführt, die von nordamerikanischen Naturvorstellungen,

11 Hannes Bergthaller, „On the Margins of Ecocriticism. A European Perspective“, in: Schmitt/ Solte-Gresser, Literatur und Ökologie (Anm. 3), S. 5-64, hier S. 10.

12 Peter Staudenmaier und Janet Biehl, Ecofascism: Lessons from the German Experience, San Francisco, AK Press, 1995.

13 Luc Ferry, Le Nouvel Ordre écologique. L'arbre, l'animal et l'homme, Paris, Grasset, 1992.

14 Axel Goodbody, „Ökologisch orientierte Literaturwissenschaft in Deutschland“, in: DürBECK/ Stовве, Ecocriticism (Anm. 2), S. 123.

152011 sehen Goodbody und Rigby grundsätzliche Unterschiede zwischen einem europäischen und einem US-amerikanischen Ecocriticism. In den USA lasse sich ein weniger ausgeprägt historisch gewachsener Bezug zwischen nationaler Identität und Natur als in Europa feststellen. Dem lässt sich die Bedeutung des Wald-Mythos für die Herausbildung der deutschen Nationalidentität entgegenhalten.

16 Schmitt/Solte-Gresser, Literatur und Ökologie (Anm. 3), S. 31.

17 Hubert ZAPF, Literatur als kulturelle Ökologie: Zur kulturellen Funktion imaginativer Texte an Beispielen des amerikanischen Romans, Berlin, De Gruyter (Konzepte der Sprach- und Literaturwissenschaft, Bd. 63), 2002; Catrin Gersdorf und Sylvia Mayer (Hg.), Beiträge zu Ökologie und Literaturwissenschaft. Natur - Kultur -Text, Heidelberg, Winter Verlag, 2005; Hubert ZAPF (Hg.), Kulturökologie und Literatur. Beiträge zu einem transdisziplinären Paradigma der Literaturwissenschaft, Heidelberg, Winter Verlag, 2008.

18 Die französischen Mitglieder des ASLE, Yves-Charles Grandjeat, Michel Granger, François Specq, sind alle Spezialisten für amerikanische Literatur und insbesondere für den nature writing Essay. Siehe Gersdorf/Mayer, Beiträge zu Ökologie und Literaturwissenschaft (Anm. 17); H. ZAPF, Literatur als kulturelle Ökologie (Anm. 17); H. ZAPF, Kulturökologie und Literatur (Anm. 17). 
klassischen Umweltschriftstellern wie Henry David Thoreau sowie literarischen Genres (autobiographisch ausgerichtete Essays und Erzählungen) geprägt waren.

\section{Deutscher und französischer Kontext: Traditionen der Umweltstudien und der Umweltbewegungen}

Sowohl in Frankreich als auch in Deutschland hat die Forschung, ohne sich auf Ecocriticism zu berufen, längst das spannende Feld der literarischen und kulturellen Repräsentationen der Mensch-Umwelt-Beziehungen untersucht. Ende der 1990er Jahre erschienen die ersten Studien zum deutschen Ecocriticism ${ }^{(19)}$, und in Frankreich eher um die Jahrtausendwende. Angesichts dieser zeitlichen Verschiebung könnte man sich fragen, weshalb der Ecocriticism in Frankreich etwas später als in Deutschland Fuß fasste. Diese leichte zeitliche Verschiebung liegt zum Teil an den unterschiedlichen geistes- und kulturgeschichtlichen Traditionen in Frankreich und Deutschland.

In Deutschland tauchte schon um die Wende vom 19. zum 20. Jahrhundert eine erste Umweltbewegung auf, die sowohl in der Romantik als auch in der Umwelt- (und Heimat) schutzbewegung, in der Lebensreformbewegung und in der aus der Anthroposophie hervorgegangenen biologisch-dynamischen Landwirtschaft (1924) ihre Wurzeln hat. In den 1970er Jahren verbreitete sich die Öko-Bewegung in Deutschland schneller als in Frankreich, zunächst aus dem Widerstand gegen die Kernenergie mit der Gründung der Grünen Partei. In den 1980er Jahren wurden vor allem aus politischen Gründen die deutschen Kulturwissenschaften von der angelsächsischen Kultur und den Cultural Studies stärker als in Frankreich beeinflusst. Man könnte auch argumentieren, dass der Ecocriticism für die Deutschen eine Möglichkeit darbot, sich wieder mit Natur zu befassen, ohne gleich verdächtigt zu werden, „Ökofaschist“ zu sein. Auf französischer Seite beschränkte sich die Auffassung der Ökologie oft auf den wissenschaftlichen Bereich oder auf einen politischen Militantismus. Interessant ist es zu bemerken, dass der Terminus „écologie“ im Dictionnaire historique de la langue française von Alain Rey so definiert ist:

„Représente un emprunt (1874) à l'allemand Ökologie, terme formé par le zoologiste et biologiste E. H. Haeckel (1834-1919) en 1866. Le mot est composé à partir du grec Oïkos - maison, habitat - et de logos - discours -, d'après économie: en français, il a pu être emprunté par l'intermédiaire de l'anglais oecology (1873), un exemple isolé de ecology ayant été relevé avant Haeckel (1852, Thoreau).“

Das Zitat zeigt, dass die Entstehung einer organischen Auffassung des Lebendigen als eines globalen Ökosystems im Imaginären der französischen Sprache eher nebenrangig und vielmehr einem von Nationalismus geprägten historischen Kontext des deutschsprachigen und angelsächsischen Raums entlehnt ist. Die Rezeption der Umweltethik Aldo Leopolds und John Baird Callicotts ${ }^{(20)}$ erfolgte in Frankreich später als in Deutschland, wo sie schon in den 1980er Jahren bekannt war und wo an

19 A. Goodbody, Literatur und Ökologie (Anm. 3).

20 John Baird Callicott, Éthique de la terre: Philosophie de l'écologie, Paris, Wildproject, 2010. Originalfassung: In Defense of the Land Ethic: Essays in Environmental Philosophy, Albany, State University of New York Press, 1989. 
verschiedenen Universitäten Lehrstühle für Umweltethik eingerichtet wurden ${ }^{(21)}$. Dies war nicht der Fall in Frankreich, wo das Buch des französischen Philosophen Luc Ferry Le Nouvel Ordre écologique (1992) jahrelang die Rezeption der Umweltphilosophien hemmte ${ }^{(22)}$. Während 2009 das Rachel Carson Center for Environment and Society in München gegründet wurde, gibt es noch kein äquivalentes internationales und interdisziplinäres Zentrum für Forschung und Bildung im Bereich der Umweltund Sozialwissenschaften in Frankreich.

Im französischen Kontext scheint keine Tradition der Romantik im Sinne einer Universalpoesie wie in Deutschland vorhanden zu sein. Die Tradition der Aufklärung und des Humanismus in Frankreich ist beherrscht von einer bestimmten Skepsis gegenüber einer engen Naturbindung, bei der man dazu neigt, „Heimatbewusstsein“ gleich mit Nationalismus und „Ökofaschismus“ zu assoziieren. Zu erwähnen wäre auch eine gewisse Skepsis gegenüber dem Natur-Begriff selbst, die die französische Philosophin und Spezialistin für Umweltethik, Catherine Larrère, als typisch französisch ansieht. Schon Descartes mochte lieber den Begriff Materie als den Begriff Natur. Der französische Sozialwissenschaftler Bruno Latour strebt seinerseits terminologisch ein „Ende der Natur“ an, das heißt, er versucht, ohne diesen Begriff zurechtzukommen. Dabei wird der Natur-Kultur-Gegensatz als Konstrukt thematisiert. Latour bevorzugt es, vom „Kollektiv“ zu sprechen. Nach Latours Actor-Network-Theory sind nicht nur menschliche Kulturen handlungsfähig, sondern auch nicht-menschliche Kulturen: auch Tiere, Bakterien, Pflanzen, Windturbinen, Maschinen sind Aktanten in einem wechselbezüglichen Netzwerk ${ }^{(23)}$. Der Material Ecocriticism betont die Wirkungskraft (agency) sowohl des Menschlichen als des Nicht-Menschlichen, sodass Natur und Kultur eng miteinander verwoben sind. Diese Verabschiedung des NaturBegriffs sei Larrère zufolge in der französischen politischen Ökologie, zum Beispiel bei Serge Moscovici oder André Gorz verbreitet und fördere nicht die Entwicklung einer Umweltethik, wie sie sich in den USA schon ab den 1950er Jahren etablierte. Larrère ihrerseits setzt sich für einen „sinnvollen Gebrauch der Natur“ ein, der die Gegensätze von Humanismus und Naturalismus überwinden solle $\mathrm{e}^{(24)}$.

Der amerikanische Professor für Politikwissenschaft Kerry Whiteside stellt zudem eine Kluft zwischen der angelsächsischen und der französischen Auffassung von Natur fest, und zwar zwischen den „noncentered ecologisms“ des französischen Denkens und den „centered ecologisms“ der englisch-amerikanischen Welt ${ }^{(25)}$. Die letzteren würden nach einem Zentrum suchen - entweder die biozentrische Natur oder

21 Konrad Otт, Jan Dierks und Lieske Voget-Kleschin (Hg.), Handbuch Umweltethik, Stuttgart, Metzler, 2016, S. 5.

22 Der Einfluss von Intellektuellen wie Jean Baudrillard und Gérard Bramoullé in den 1990er Jahren wäre noch als Hemmschuh zu erwähnen.

23 Bruno Latour, Das Parlament der Dinge - Für eine politische Ökologie, Berlin, Suhrkamp, 2013 (Aus dem Französischen von Gustav Roßler). Originalfassung: Politiques de la nature. Comment faire entrer les sciences en démocratie, Paris, La Découverte, 1999.

24 Catherine Larrère, Du bon usage de la nature. Pour une philosophie de l'environnement, Paris, Flammarion (Champs Essais), 1997.

25 Kerry H. Whiteside, Divided Natures: French Contributions to Political Ecology, Cambridge, MA, The MIT Press, 2002. 
den anthropozentrischen Menschen -, um ein absolutes Wertesystem zu gründen, während die französischen Theoriker der Ökologie (René Dumont, Serge Moscovici, Edgar Morin, Michel Serres, Bruno Latour, Emmanuel Mounier, Denis Duclos, JeanPaul Deléage, Alain Lipietz) eher versuchen, die Natur-Kultur-Problematik zu verfeinern und die Prozesse zu beschreiben, die Natur und menschliche Identität verbinden.

Es lassen sich also unterschiedliche Referenztexte und Traditionslinien nachweisen. Im deutschen Kontext zeigt sich eine breite Rezeption von der romantischen (Tieck, Novalis) und Goetheschen Naturphilosophie, von den Naturwissenschaften und der Ökologie als Wissenschaft (Alexander von Humboldt, Ernst Haeckel), von der Phänomenologie (Heidegger, Gernot Böhme), der Kritischen Theorie (Benjamin, Adorno), der Verantwortungsethik (Jonas), der Umweltethik (Klaus Michael Meyer-Abich, Dieter Birnbacher, Günther Altner), der Systemtheorie (Luhmann) und der Gesellschaftstheorie (Ulrich Beck). In Frankreich hingegen dienen eher die Arbeiten aus dem Bereich der Ökologie (Bruno Latour, Michel Serres), der Anthropologie (Philippe Descola), der Soziologie (Roger Caillois) und Philosophie (Gaston Bachelard, Pierre Hadot, Félix Guattari, Catherine Larrère) als Bezugspunkte, wobei die Naturästhetik und Umweltästhetik ${ }^{(26)}$ sowohl in Frankreich als auch in Deutschland eine wichtige Rolle spielen.

Wie bei dem Vergleich zwischen Europa und USA könnte argumentiert werden, dass in Frankreich die Auseinandersetzung mit Mensch-Umwelt-Beziehungen eine lange Tradition der philosophischen, ökologischen, anthropologischen und soziologischen Naturforschung aufweist ${ }^{(27)}$, so dass von einem verspäteten Interesse nicht die Rede sein kann. Im Rahmen einer vergleichenden Studie ist es aufschlussreich, die spezifischen Ansätze auf beiden Seiten zu skizzieren und die Bezugspunkte aufzuzeigen.

\section{Écocritique/Ecocritism: zwei unterschiedliche Entwicklungen im französischen und deutschen Kontext}

\section{Spezifische Ansätze der französischen „écocritique“}

In Frankreich hat sich die écocritique eher als literaturwissenschaftliches Forschungsgebiet denn als Bereich der Kulturwissenschaften entwickelt, und zwar in Richtungen, die grob als „écopoétique“, „écocritique comparée“ (vergleichende Ökokritik), „épistémocritique" und Forschungen zu Literatur und Raum umrissen werden können. Der 2018 erschienene Aufsatz der Kulturgeographen Nathalie Blanc und Denis Chartier sowie des Literaturwissenschaftlers und Amerikanisten Thomas Pughe, „Littérature et écologie: vers une écopoétique“ („Literatur und Ökologie. Zu einer Ökopoetik“) hebt die besondere Rolle der politischen und poetischen („po-ethischen“) Dimension des Ecocriticism im französischen Kontext hervor. Der Begriff „écopoétique“ entstand in Belgien und Frankreich nicht nur als eine französische Übersetzung des englischen Begriffs „ecopoetics“. Dieser grundsätzlich interdisziplinäre Ansatz versteht sich

26 Gernot Böнme, Für eine ökologische Naturästhetik, Frankfurt am Main, Suhrkamp, 1989; Jost HeRMAND, Im Wettlauf der Zeit: Anstöße zu einer ökologiebewussten Ästhetik, Berlin, Edition Sigma, 1991; Nathalie Bourriaud, Esthétique relationnelle, Dijon, Presses du réel, 2001; Nathalie Blanc, „Esthétique de la nature“, in: Aurélie Choné, Isabelle Hajek und Philippe Hamman (Hg.), Guide des Humanités environnementales, Villeneuve d'Ascq, Presses universitaires du Septentrion, 2016, S. 157-164. 
vielmehr als Brücke von einer Umweltpolitik zu einer Naturästhetik, die Interaktionen zwischen Mensch und Umwelt in Formen wie „land art“ oder poetischer Performance künstlerisch gestaltet. Eine Ökokritik, die innerhalb der literarischen Studien einen legitimen Platz finden möchte, stehe den Autoren zufolge vor der Herausforderung, sich über die „ökologische Arbeit des literarischen Schreibens “ ${ }^{(28)}$ Gedanken zu machen, über die (Neu)erfindung des Natur-Begriffs innerhalb der Sprache und der poetischen Imagination, was an die Rolle der Literatur als Kulturökologie erinnert, wie sie insbesondere von Hubert Zapf in Deutschland hervorgehoben wurde ${ }^{(29)}$. Dem Professor für französische Literatur an der Universität Gand (Belgien) Pierre Schoentjes zufolge gilt es in der „écopoétique“ nicht nur, ältere Autoren neu zu lesen (Julien Gracq) oder neu zu entdecken (Pierre Gascar), sondern die Texte neuer Autoren (Hubert Mingarelli, André Bucher, Pascal Wick, Jean-Loup Trassard) unter der Berücksichtigung ihrer Beziehung zur Natur und Umwelt zu erforschen ${ }^{(30)}$. Die besondere Rolle der "écopoétique“ im französischen Kontext kommt deutlich in der Benennung des ersten diesbezüglichen Vernetzungsversuchs in Frankreich zum Ausdruck, und zwar durch die Initiative der Universität Perpignan, 2016 ein „Atelier de recherche en écocritique et écopoétique“ zu gründen, und darin alle Forschenden, die zur „écocritique“ und „écopoétique“ in Frankreich arbeiten, zu vernetzen ${ }^{(31)}$. Von der Bedeutung der poetischen Dimension zeugt auch das 2018 gegründete Kollektiv ZoneZadir, dessen Name auf das Akronym „ZAD“(32) anspielt und damit auch stark die politische Dimension unterstreicht: „Le collectif ZoneZadir est un réseau de chercheurs en littérature cherchant à promouvoir l'écopoétique“(33). Der Begriff „écopoétique“ wird explizit verwendet, um „sich etwas vom amerikanischen Ecocriticism abzugrenzen“ “(34) und auf Fragen der Poetik zu fokussieren.

Nach seinem ersten Buch Amerikanische Literatur und Ökologie versuchte der französische Literaturwissenschaftler und Spezialist für nordamerikanische Literatur, Alain Suberchicot, in seinem zweiten Buch Literatur und Umwelt. Zu einer vergleichenden Ökokritik ${ }^{(35)}$ den Ansatz des Ecocriticism aus dem nordamerikanischen Kontext

28 Nathalie Blanc, Thomas Pughe und Denis Chartier, „Littérature et écologie: vers une écopoétique“, Écologie et politique, 36 (2008), S. 1-12 (meine Übersetzung).

29 H. ZAPF, Literatur als kulturelle Ökologie (Anm. 17).

30 Pierre Schoentjes, „Texte de la nature et nature du texte. Jean-Loup Trassard et les enjeux de l'écopoétique en France“, Poétique, 164/4 (2010), S. 477-494. Siehe auch Pierre Schoentjes, Ce qui a lieu: essai d'écopoétique, Marseille, Wildproject, 2015.

31 Ecopoetics Perpignan, Atelier de recherche en écocritique et écopoétique, ecopoeticsperpignan.com.

32 Der französische Ausdruck „zone à défendre“ (ZAD) ist ein militanter Neologismus, der eine Art Protest-Besetzung (meistens unter freiem Himmel) kennzeichnet und ein politisches Ziel verfolgt, meistens gegen ein Bauprojekt (Flughafen, industrielle Bauernhöfe usw.).

33 zonezadir.hypotheses.org.

34 zonezadir.hypotheses.org: „Ce n'est qu'au début des années 2000 que des travaux ont tenté de rendre visible cette discipline en France, employant alors explicitement le terme ,écopoétique', afin de se distinguer légèrement de l'écocritique américaine apparue à la fin des années 70, mais devenue réellement visible dans les années 90. Ce qui est en jeu dans ce glissement, c’est la volonté de faire de l'écopoétique, une discipline recentrée autour des questions poétiques“ (meine Übersetzung des Passus in Kursivschrift).

35 Alain Suberchicot, Littérature et environnement. Pour une écocritique comparée, Paris, Honoré Champion (Unichamp-Essentiel), 2012. 
herauszulösen und in der vergleichenden Literaturwissenschaft zu verankern. Die französischen Amerikanisten (Granger, Pughe, Suberchicot...) untersuchen das ökologische Schreiben anders als ihre amerikanischen Kollegen: „L'influence de l'héritage français - l'ère du soupçon, les Nouveaux Romanciers, les écrits de Deleuze, Derrida, Foucault et Lacan, entre autres - rend impossible le retour au réalisme simple“(36). Suberchicots Essay spricht sich gegen die Idee aus, es gäbe nur eine umweltorientierte Literatur im angelsächsischen Raum und Frankreich hätte keine. Der Autor beleuchtet viele Texte aus der französischen Literatur wie jene von Jacques-Henri Fabre, Victor Segalen, Marguerite Duras und Jean-Marie Le Clézio im Hinblick auf die Beziehungen zwischen Mensch und Umwelt. Er kritisiert auch einige Texte aus der nordamerikanischen Fachliteratur, z.B. Texte von wichtigen Vertretern des amerikanischen nature writing wie Edward Abbey und Annie Dillard, die ihm zu „oberflächlich“, zu „lyrisch“ und sozial fremd erscheinen. Ihn interessieren vielmehr nicht-spezialisierte Texte, die nicht den Erwartungen eines main stream Publikums entsprechen und die eher dazu tendieren, Umweltfragen implizit zu behandeln. Suberchicot richtet seine Aufmerksamkeit dabei auf die Verbindung zwischen Literatur und sozialpolitischen Fragen und verweist unter anderem auf eine für Julien Gracq typische Verwobenheit zwischen Geschichte und Landschaft an.

Stéphanie Posthumus, Literaturwissenschaftlerin aus Montréal, schlägt einen anderen Ansatz vor, um zur Entwicklung einer französischen écocritique beizutragen. Wie Suberchicot möchte sie die Ökokritik von der sprachlichen Hegemonie des Englischen befreien. Die französische Ökokritik sollte sich ihrer Meinung nach auf französische Natur-Vorstellungen und Denktraditionen stützen. Deswegen verwendet sie die französischen Theorien zur Ökologie, unter anderem die „écosophie“ von Félix Guattari, die „nature-culture“ von Bruno Latour und den „Naturvertrag“ („contrat naturel“) von Michel Serres, um Themen wie Natur, Umwelt und Landschaft in zeitgenössischen französischen Erzählungen zu analysieren (z.B. von Jean-Christophe Rufin, Michel Houellebecq, Marie Darrieussecq und Michel Tournier). Um eine französische Ökokritik zu begründen, beruft sie sich vor allem auf Michel Serres ${ }^{(37)}$ wegen der literarischen Qualität und der interdisziplinären Herangehensweise in seinen Schriften ${ }^{(38)}$. Posthumus lobt die poetische und metaphorische Dimension des Naturvertrags und verweist auf drei Figuren, die für die Ökokritik von großer Bedeutung sein könnten: den Bauer, den Seemann und den Wanderer. Die zwei ersten Figuren

36 Stéphanie Posthumus, „État des lieux de la pensée écocritique française“, Ecozona, 1/1 (2010), S. 148, core.ac.uk/download/pdf/58910791.pdf.

37 Stéphanie Posthumus, „Vers une écocritique française: le contrat naturel de Michel Serres“, Mosaic: An Interdisciplinary Critical Journal, 44/2 (2011), S. 85-100, hier S. 93-94: „L'écocritique s'appuiera sur le pouvoir poétique du contrat naturel qui permet d'imaginer de nouveaux mondes. Plutôt que de réduire le texte à une thèse écologique - interprétation trop réductrice allant à l'encontre de la pluralité de la pensée chez Serres - l'écocritique cherchera à déterminer dans quelle mesure les différentes réalités représentées dans le texte littéraire bouleversent nos idées préconçues, nos rapports trop limités avec la terre. En examinant les multiples réseaux sémantiques des espaces naturels, ruraux, urbains, mondiaux, etc., dans le texte littéraire, l'écocritique mettra en lumière les multiples scénarios imaginaires dont l'être humain pourra s'inspirer pour trouver des solutions aux problèmes écologiques."

38 Michel Serres, Le contrat naturel, Paris, Seuil, 1990 (deutsche Übersetzung von Hans-Horst Henschen: Der Naturvertrag, Frankfurt am Main, Suhrkamp, 1994). 
veranschaulichen ihrer Meinung nach eine französische Umweltethik und die dritte eine ökologische Herangehensweise, die aber keine cartesianische und rationalistische Methode sein soll. Die zwei letzteren sind auch für die Geopoetik (auf die ich später zu sprechen komme) von großer Bedeutung.

Da der Ecocriticism sich mit Themen beschäftigt, die nicht neu sind, fällt es den Forschenden ziemlich leicht, sich auf Vorläufer aus ihren jeweiligen Denktraditionen zu beziehen. Als „Vorläufer“ ökokritischer Forschungen in Frankreich spielen Serres, Bachelard und Caillois eine besondere Rolle, insofern sie die Bedeutung des poetischen Imaginären in den Mensch-Umwelt-Beziehungen hervorheben - was meines Erachtens einen weiteren spezifischen Ansatz des Ecocriticism im französischen Kontext bildet. Wie Clare Esposito zeigt, könnte Caillois’ Projekt der „diagonalen Wissenschaften“ den Ökokritikern von Nutzen sein: „Le courant écocritique ne pourrait que gagner à puiser dans l'œuvre de Caillois, en explorant les possibilités d'une mise en relation de la science et de la philosophie de l'écologie avec des questions d'esthétique littéraire“(39). Caillois betont das Spezifische der poetischen Imagination: „La poésie n'est possible que s'il existe un fonds commun objectif de l'imagination “(40). Ihm zufolge sei das künstlerisch Imaginäre dazu fähig, tief liegende Entsprechungen wieder auftauchen zu lassen: „L'attention au monde est devoir de la poésie“(41). Auf die Bedeutung der Aufmerksamkeit verweist auch der französische Literaturwissenschaftler Yves Citton in seiner „Ökologie der Achtsamkeit “(42). Die Betonung der achtsamen Wahrnehmung des Dichters scheint mir auch mit der Herangehensweise des französischen Literaturwissenschaftlers Jean-Pierre Richard verwandt ${ }^{(43)}$. Die Erweiterung der Imaginationsübungen auf nicht-menschliche Bereiche stellt eine große Herausforderung dar. Aus diesem Bemühen ergibt sich die Möglichkeit einer Auflösung der Unterscheidung zwischen dem Menschlichen und dem Nicht-Menschlichen, wie auch das Auffinden von erstaunlichen Entsprechungen und Resonanzen zwischen weit entfernten Bereichen, zum Beispiel zwischen Kunstwerken und Mineralien. Caillois ist immer davon ausgegangen, dass das Menschliche nicht von seinem eigenen geschlossenen System abhängt. Die Formen einer nicht-figurativen Kunst, welche die Ausnahme „Mensch“ am deutlichsten belegen soll, werden neben mikroskopische Aspekte von Kristallen, Plankton und Rinden gestellt, damit das Menschliche und das Nicht-Menschliche als „Zwei Hemisphären der Ästhetik“(44) empfunden werden. Aus den Gemeinsamkeiten zwischen weit entfernten Formen lasse sich folgern, dass „ein wirklich formloses Werk undenkbar ist: oder eher dass es als nicht figurativ vorkommt, solange man die Realität, die ihr als Modell hätte dienen können, nicht neben sie gestellt hat “(45).

Clare Sibley-Esposito, „Caillois sur les chemins de l'écocritique“, Littératures, 68 (2013), S. 141-160, journals.openedition.org/litteratures/108.

40 Roger Caillois, Cases d'un échiquier, Paris, Gallimard, 1970, S. 249.

41 Ebd., S. 253.

42 Yves Citton, Pour une écologie de l'attention, Paris, Seuil, 2014.

43 Jean-Pierre Richard, Littérature et sensation, Paris, Seuil, 1954.

44 R. Caillois, Cases d’un échiquier (Anm. 40), S. 184: „deux hémisphères de l'esthétique“ (meine Übersetzung).

45 Ebd., S. 184: „[...] une œuvre vraiment informelle est inconcevable; ou plutôt [...] elle ne paraît non figurative que tant qu'on n'a pas placé à côté d’elle la réalité qui aurait pu en être le modèle“ (meine Übersetzung). 
Caillois schreibt: „La poésie ne consiste pas à mettre en vers les résultats du savoir elle est elle-même méthode et savoir “(46). Dies genau bildet die Prämisse der „épistémocritique“, auf die ich kurz eingehen möchte, weil sie meines Erachtens einen weiteren spezifischen Ansatz der französischen Ökokritik darstellt, obwohl die wechselseitige Beziehung zwischen Literatur und Wissen auch in Deutschland im Zentrum der gegenwärtigen Kulturwissenschaften steht ${ }^{(47)}$. Die französische „écocritique“ ist mit einer „épistémocritique “(48) der Natur eng verbunden, wie sie unter anderem Laurence Dahan-Gaida ${ }^{(49)}$ und Gisèle Séginger ${ }^{(50)}$ vertreten. Wie sonst können wohl die Beziehungen eines literarischen Textes zur physischen Umgebung untersucht werden, ohne das Wechselspiel von Literatur und Naturwissen(schaften), Naturvorstellungen und Naturdarstellungen miteinzubeziehen? In der „épistémocritique de la nature“ geht es darum, die Austauschprozesse zwischen literarischen und nicht-literarischen Diskursen über Natur und Umwelt zu untersuchen, die Rolle der Literatur als Wissensvermittlung zu verstehen, das sprachlich verfasste Wissen über Natur, das die Literatur bereithält, herauszuarbeiten und kritisch zu interpretieren.

Das Verhältnis zur natürlichen Umwelt, um das es im Ecocriticism geht, setzt die räumliche Dimension der physischen Umgebung voraus, was auch für die französische Ökokritik charakteristisch ist. Wenn die französische „écocritique“ sich am Schnittpunkt der Forschungen zu Literatur und Raum bewegt, liegt das sicher daran, dass der Fokus auf das Räumliche in Frankreich weniger mit geopolitischen Fragen verknüpft ist als in Deutschland, wo der sogenannte spatial turn auf eine gewisse Skepsis gestoßen ist:

„Die massiven Vorbehalte gegenüber geopolitischen Ansätzen gehen zurück auf die nationalsozialistische Ideologisierung und Funktionalisierung des Raumkonzepts für die Propaganda und Kriegspolitik des zweiten Weltkriegs, wie sie sich in einer rassistischen Blut-und-Boden-Ideologie und in der Zielvorstellung einer gewaltsamen Erweiterung des Lebensraums im Osten für ein ,Volk ohne Raum 'verhängnisvoll niedergeschlagen hat “(51).

In Frankreich beziehen sich auch aktuelle Lyriktheorien auf die Kategorie des Raumes, insbesondere auf Konzepte der „géocritique“, der „pensée-paysage“ und der "géo-poétique“. Als Vorläufer könnte Gaston Bachelard genannt werden, der mit seiner Poetik des Raums Bilder intimer Räumlichkeit wie das Haus, den Schlupfwinkel und die Höhle untersucht hat ${ }^{(52)}$. Der griechische Begriff „oikos“ umfasst ein breites

46 Ebd., S. 251.

47 Roland Borgards, Harald Neumeyer, Nicolas Pethes und Yvonne Wübben (Hg.), Literatur und Wissen: Ein interdisziplinäres Handbuch, Stuttgart, Metzler Verlag, 2013 (siehe Beitrag von Ursula K. Heise „Ecocriticism“).

48 Michel Pierssens, Savoirs à l'ouvre: Essais d'épistémocritique, Villeneuve d'Ascq, Presses universitaires du Septentrion, 1990.

49 Laurence DAHAN, „Nature en culture: l'approche épistémocritique de la Nature“, in: CHONÉ/HAJEK/ Hamman, Guide des Humanités environnementales (Anm. 26), S. 173-182.

50 Gisèle SÉGinger (Hg.), „Flaubert, les sciences de la nature et de la vie“, Flaubert. Revue critique et génétique, 13 (2015), flaubert.revues.org/2406.

51 Doris Bachmann-Medick, Cultural Turns: Neuorientierungen in den Kulturwissenschaften (2006), Hamburg, Rowohlt (rowohlts enzyklopädie, 55675), 2014 (5. Aufl.), S. 286.

52 Gaston Bachelard, La poétique de l'espace, Paris, Presses universitaires de France, 1957; Die Poetik des Raumes, Frankfurt am Main, Fischer, 1987. 
Bedeutungsfeld, wo sich Vorstellungen von „Behausung“ entfalten können. „Oikos“ kann sich vom bloßen Gebäude zum Wohnen und Arbeiten bis auf die ganze Erde, ja auf den Kosmos erstrecken. In Anlehnung an Hölderlin ${ }^{(53)}$, der stark von Heidegger rezipiert wurde und dessen Rezeption in Frankreich auf breites Interesse stieß, geht es darum, mittels der poetischen Sprache einen „oikos“, eine Wohnstätte, zu errichten. Diese in Frankreich fest etablierte Tradition der Literatur als „oikos“ wird derzeit von französischen Dichtern wie Philippe Jaccottet ${ }^{(54)}$, Michel Deguy oder Jean-Claude Pinson vertreten:

„[...] une approche aussi bien théorique que poétique (en acte) de l'écologie poétique peut nous ramener au centre des passions relationnelles entre le sujet vivant et le monde, à la recherche d'un monde commun à partager à partir d'un constat de perte ou de menace pour la survie. [...] Capacité de mise en relation (,symbolec, , articulation'), ouverture panoptique au Tout du monde (,comprendre', ,affaire de vision'), la Maison-Littérature est bien de ce monde, dans le monde, mais aussi réancrage permanent au monde“(55).

Das, was Jean-Marie Schaeffer „écologie des études littéraires“ nennt, ist eine Form des aufmerksamen Umgangs mit der Sprache, die an die „Ökologie der Achtsamkeit“ von Yves Citton ${ }^{(56)}$ anknüpft, und die mit Berbeli Wannings Vorhaben, Kulturökologie und Literaturdidaktik zu verbinden, verwandt ist.

Öko- und geokritische Ansätze legen gemeinsam den Akzent auf die Beziehung zwischen Mensch und Umwelt/Erde in literarischen Werken ${ }^{(57)}$. Sie sind geo- und ökozentriert. Wohl haben sich bis heute Geokritik, Geopoetik, Ökopoetik und Ökokritik relativ unabhängig voneinander entwickelt. Der französische Terminus géopoétique wurde von zwei Dichtern geprägt, Michel Deguy und Kenneth White. Das Institut international de géopoétique wurde von White außerhalb eines universitären Rahmens 1989 in Paris gegründet, obwohl der schottisch-französische Essayist lange einen Lehrstuhl für Dichtung des 20. Jahrhunderts an der Sorbonne innehatte. White bezeichnet sich selbst als „intellektuellen Nomaden“. Sein Institut hat sich zur Aufgabe gesetzt, die Zusammenarbeit von Schriftstellerinnen und Schriftstellern, Kunstschaffenden, Lehrenden, Studierenden, Berufstätigen, Wissenschaftlerinnen und Wissenschaftlern transdisziplinär zu fördern. In seinem Essay Éléments de géopoétique von 1987 entwirft White die Geopoetik als ein Projekt an der Grenze zwischen Poesie und Wissenschaft, zwischen konkreter Geographie und „geistigem Raum“. In der Vermischung von wissenschaftlichem und poetischem Diskurs bei der Erdentdeckung, -erkundung und -beschreibung sucht er nach Texten und Projekten, in denen Dichter zu Geographen oder Geographen zu Dichtern werden (als Beispiele nennt er Rimbaud und Whitman).

53 Ich verweise hier auf Friedrich Hölderlins berühmte Verse aus seinem Gedicht „In lieblicher Bläue“: "dichterisch wohnet der Mensch auf dieser Erde“.

54 Siehe Peter Schnyder, „Poétique de la nature“, in: Choné/Hajek/Hamman, Guide des Humanités environnementales (Anm. 26), S. 123-134.

55 Martine Boyer-WeinmanN, „Comment, l'écologie littéraire peut-elle raviver la relation aux œuvres et à leur transmission?", Études de lettres, 1 (2014), S. 151-164.

56 Y. Citton, Pour une écologie de l'attention (Anm. 42).

57 Stephanie Posthumus und Rachel Bouvet, „Eco- and Geo-Approaches in French and Francophone Literary Studies“, in: Hubert ZAPF (Hg.), Handbook of Ecocriticism and Cultural Ecology, Berlin/Boston, De Gruyter, 2016, S. 385-412. 
Im Zuge des sogenannten spatial turn wurde ein anderer Ansatz, diesmal innerhalb des universitären Kontexts gegründet, und zwar die „géocritique“(58). Dieser Begriff wurde erstmals 2000 durch den französischen Komparatisten Bertrand Westphal eingeführt und dann grundlegend in seinem Buch La Géocritique. Réel, Fiction, Espace (2007) dargelegt. Westphal definiert seine Geokritik als Methode, die das Verhältnis von Literatur und geographischem Raum untersucht. Die ausdrückliche Bezugnahme auf den geographischen Raum ist dabei zentral. Geokritische Studien im Sinne Westphals bedeuten, dass eine einzelne Stadt oder eine Region unter Berücksichtigung einer möglichst großen Zahl literarischer Zeugnisse untersucht wird, d.h. sie ist multifokal. Sie befasst sich auch mit der sinnlichen Wahrnehmung von Räumen (Polysensorialität) und erforscht „synästhetische Landschaftsbilder“. Untersucht wird schließlich die Intertextualität von Räumen: Wie textuell beladen ist der beschriebene Raum, die beschriebene Landschaft?

In La Pensée-Paysage. Philosophie, arts, littérature ${ }^{(59)}$ unterstreicht der französische Literaturwissenschaftler Michel Collot das aktuelle Interesse an Landschaft in Europa: Dieses Interesse sei keine Mode, sondern entspreche vielmehr einer tiefgreifenden geschichtlichen Entwicklung der Mentalitäten. Die Urbanisierung und die Umweltzerstörung würden die Landschaft bedrohen und sie dadurch kostbar machen. Die Landschaft würde das Bedürfnis der Menschen widerspiegeln, an eine sinnliche Wahrnehmung der Umwelt neu anzuknüpfen. Sie ermöglicht Collot zufolge ein anderes Denken, das sogenannte „Landschaftsdenken“, dessen Ausprägungen er in Philosophie, Kunst und Literatur untersucht ${ }^{(60)}$.

Die Komplementarität und Diversität dieser geo- und ökokritischen Ansätze besitzt meines Erachtens ein großes Potential ${ }^{(61)}$. Jeder Ansatz arbeitet mit seinen eigenen Konzepten und Methoden: Landschaften, Reisen, Wohnen, Beziehungen zwischen Mensch und Globus (im Falle der Geopoetik); Polysensorialität, Multifokussierung, Stratigraphie, Beziehungen zwischen Menschen und Räumen (im Falle der Geokritik); Natur, Kunst, Performance, Ökologie, „Sense of Place“ (im Falle der Ökopoetik); Umweltbewusstsein, politische Ökologie, Verhältnis von Mensch und nichtmenschlicher Natur (im Falle der Ökokritik). Es kann extrem fruchtbar sein, den literarischen Text aus der Perspektive mehrerer geo- und ökokritischen Ansätze zu erforschen. Eine solche Interpretationsweise literarischer Texte bildet vielleicht kein neues Paradigma in der Ökokritik-Forschung, erscheint mir immerhin als ein interessanter und spezifischer Beitrag der französischen Ökokritik zu diesem Forschungsfeld. Ich möchte zum Schluss darauf hinweisen, dass das seit 2014 bestehende Projekt EcoLitt ${ }^{(62)}$, das

58 Bertrand Westphal, La Géocritique. Réel, fiction, espace, Paris, Minuit, 2007; Bertrand Westphal, La cage des méridiens. La littérature et l'art contemporain face à la globalisation, Paris, Minuit, 2016.

59 Michel Collot, La pensée-paysage. Philosophie, arts, littérature, Arles, Actes Sud/ENSP (coll. „Paysage“), 2011.

60 Siehe auch Pascale SAlinier, Paysages habités. Petite anthologie de littérature, s.l., Éditions de l'Escarmouche, 2011.

61 Siehe auch Eric Prieto, „Geocriticism Meets Ecocriticism: Bertrand Westphal and Environmental Thinking“, Épistémocritique, 9 (2011), epistemocritique.org/geocriticism-meets-Ecocriticismbertrand-westphal-and-environmental-thinking/.

62 „Par une mutualisation des compétences des chercheurs comparatistes de différentes spécialités linguistiques et littéraires, EcoLitt vise à étayer la recherche dans le domaine encore méconnu en 
vom Forschungszentrum CERIEC (Centre d'Études et de Recherches sur Imaginaire, Écriture, Culture) der Universität Angers und von der Region Pays de la Loire drei Jahre lang finanziert wurde, die verschiedenen Ansätze der „écocritique“ im französischen Kontext verbindet.

\section{Spezifische Ansätze des deutschen Ecocriticism}

Man könnte sich fragen weshalb die deutschen Forschenden den angelsächsischen Begriff „Ecocriticism“ direkt übernommen haben, während ihre französischen Kolleginnen und Kollegen den Begriff „écocritique“ geprägt haben. Teilweise wird dieser auch im Deutschen verwendet, möglicherweise importiert aus dem Französischen ${ }^{(63)}$. Diese unterschiedlichen Bezeichnungen verweisen auf grundlegende Unterschiede zwischen dem deutschen und dem französischen Kontext. Der Hauptunterschied besteht wahrscheinlich in der kulturwissenschaftlichen Orientierung des deutschen Ecocriticism und im Einfluss von Pionierarbeiten englischsprachiger Germanisten wie Axel Goodbody ${ }^{(64)}$ (Großbritannien) und Kate Rigby ${ }^{(65)}$ (Australien). Goodbody nennt z.B. Kulturwissenschaftler wie Hartmut Böhme ${ }^{(66)}$ und Jost Hermand ${ }^{(67)}$ als Vorläufer des Ecocriticism, obwohl gerade diese beiden den Begriff nicht verwenden. Böhmes Ästhetik der Natur hat aber den deutschen Ecocriticism stark beeinflusst. In mehreren Veröffentlichungen beschäftigt sich der Professor für Kulturtheorie und Mentalitätsgeschichte mit dem Verhältnis des Menschen zur Natur ${ }^{(68)}$. Böhmes Werk könnte mit den Arbeiten des französischen Historikers Alain Corbin verglichen werden, der im Sinne der Mentalitätsgeschichte Lucien Febvres verschiedene Themen wie das Verhältnis des Menschen zum Ufer, die Lautlandschaft auf dem französischen Land im 19. Jahrhundert, und aktuell die Kulturgeschichte des Regens und des Grases behandelt hat, wobei Corbin als Historiker der sinnlichen Wahrnehmung den Akzent auf die Geschichte der Emotionen legt ${ }^{(69)}$. Nach den Sammelbänden von Peter Morris-Keitel

France de l'écocritique et de l'analyse des rapports entre la littérature et les préoccupations environnementales. L'ambition du projet est de faire progresser la recherche, par un travail transdisciplinaire régional, sur des problématiques littéraires encore peu reconnues en France“, ecolitt.univ-angers.fr/fr/ index.html.

63 Ursula K. HeIse, „Ökokritik/Ecocriticism“, in: Ansgar NüNnING (Hg.), Metzler Lexikon für Literaturund Kulturtheorie, Stuttgart, Metzler, 2001, S. 155-156.

64 Axel Goodbody, Nature, Technology and Cultural Change in Twentieth-Century German Literature. The Challenge of Ecocriticism, Basingstoke, Palgrave, 2007.

65 Kate Rigby, Topographies of the Sacred, The Poetics of Place in European Romanticism, Charlottesville, University of Virginia Press, 2004.

66 Hartmut Вӧнме, „Literaturwissenschaft in der Herausforderung der technischen und ökologischen Welt“, in: Ludwig JäGER und Bernd Switalla (Hg.), Germanistik in der Mediengesellschaft, München, Verlag Wilhelm Fink, 1994, S. 63-79.

67 Jost Hermand, „Literaturwissenschaft und ökologisches Bewusstsein. Eine mühsame Verflechtung“, in: Anne Bentfeld und Walter Delabar (Hg.), Perspektiven der Germanistik, Opladen, Westdeutscher Verlag, 1997, S. 106-125.

68 Hartmut Böнme, Kulturgeschichte des Wassers, Frankfurt am Main, Suhrkamp, 1988; Hartmut Вӧнме, Gernot Вӧнме, Feuer Wasser Erde Luft. Kulturgeschichte der Naturwahrnehmung in den Elementen, München, C.H. Beck, 1996.

69 Alain Corbin, Le territoire du vide, Paris, Flammarion, 1990; Les cloches de la terre, Paris, Albin Michel, 1994; Histoire buissonnière de la pluie, Paris, Flammarion, 2017; La fraîcheur de l'herbe. 
und Michael Niedermeier einerseits ${ }^{(70)}$, Gerdorf und Mayer andererseits ${ }^{(71)}$, wurde die erste deutschsprachige Einführung zum Ecocritism von Gabriele Dürbeck und Urte Stobbe herausgegeben ${ }^{(72)}$. Sie stellt unter anderem die spezifischen Ausprägungen eines deutschen Ecocriticism vor. 2016 folgte Benjamin Bühlers Monographie, die den deutschen Ecocriticism als „Paradigma für eine kulturwissenschaftlich erweiterte Germanistik“(73) vorstellt. In Deutschland lassen sich grob folgende Grundrichtungen des Ecocriticism unterscheiden: Kultur- und Literaturökologie, ökologische Genres und Schreibmodi, Cultural Animal and Plant Studies, Anthropozän-Debatte.

Der deutsche Ecocriticism umfasst kulturwissenschaftliche Fragestellungen und Perspektiven, wie zum Beispiel die Kulturökologie des Amerikanisten Hubert Zapf, wobei diese nicht gleichbedeutend dem französischen Begriff „écologie culturelle“ ist, der wiederum auf den amerikanischen Anthropologen Julian Steward zurückgeht (cultural ecology). Der deutsche Literaturwissenschaftler betrachtet seinerseits die Literatur als kulturelle Ökologie und erforscht die literarische Kreativität aus textethischer Perspektive ${ }^{(74)}$. Der Literatur- und Kulturwissenschaftler Stefan Hofer hingegen erforscht die „Ökologie der Literatur“ aus einer systemtheoretischen Perspektive, indem er das Paradigma des Ecocriticism mit Niklas Luhmanns Systemtheorie auf eine tragfähige theoretische Grundlage zu stellen versucht ${ }^{(75)}$. Seit 10 Jahren versucht die Germanistin Berbeli Wanning Kulturökologie und Literaturdidaktik, Didaktik und Ecocriticism, Bildung und Nachhaltigkeit zu verbinden ${ }^{(76)}$. Man könnte zu diesen Forschungen eine Parallele zu den Arbeiten von Jean-Marie Schaeffer ${ }^{(77)}$, Martine Boyer-Weinmann ${ }^{(78)}$ und Yves Citton ${ }^{(79)}$ ziehen.

Der deutsche Ecocriticism beschäftigt sich neuerdings auch intensiv mit ökologischen Genres und Schreibmodi ${ }^{(80)}$ wie Utopie/Dystopie, Robinsonade, Science Fiction,

Histoire d'une gamme d'émotions de l'Antiquité à nos jours, Paris, Fayard, 2018. Corbins Einfluss auf die „écocritique“ erfolgt vor allem durch seine Analysen zur Landschaft, d.h. eher im Rahmen der Orientierung „Literatur und Raum“. Siehe Alain Corbin, L’homme dans le paysage, Paris, Seuil, 2001.

70 Peter Morris-Keitel und Michael Niedermeier, Ökologie und Literatur, New York, Peter Lang, 2000.

71 Catrin Gersdorf und Sylvia Mayer (Hg.), Beiträge zu Ökologie und Literaturwissenschaft, Heidelberg, Winter Verlag, 2005.

72 Dürвеск/Stовве, Ecocriticism. Eine Einführung (Anm. 2).

73 Benjamin BüHLER, Ecocriticism: Grundlagen - Theorien - Interpretationen, Stuttgart, Metzler, 2016, siehe „Einführung“.

74 H. ZAPF, Literatur als kulturelle Ökologie (Anm. 17); H. ZAPF, Handbook of Ecocriticism and Cultural Ecology (Anm. 57).

75 Stefan Hofer, Die Ökologie der Literatur: eine systemtheoretische Annäherung. Mit einer Studie zu Werken Peter Handkes, Bielefeld, transcript, 2007.

76 Berbeli Wanning und Axel Goodbody (Hg.), Wasser - Kultur - Ökologie. Konstanten und Wandel in der sozialen und kulturellen Bedeutung des flüssigen Elements, Göttingen, V\&R unipress, 2008.

77 Jean-Marie Schaeffer, Petite écologie des études littéraires, pourquoi et comment étudier la littérature?, Vincennes, Éditions Thierry, 2011.

78 M. Boyer-Weinmann, „Comment, l’écologie littéraire“ (Anm. 55).

79 Y. Citton, Pour une écologie de l'attention (Anm. 42).

80 Evi Zемалек (Hg.), Ökologische Genres. Naturästhetik - Umweltethik - Wissenspoetik, Göttingen, Vandenhoeck \& Ruprecht, 2018. Diesen Aspekt behandelt im gleichen Band ausführlich der Aufsatz von Evi Zemanek, deswegen werde ich mich in diesem Rahmen nicht weiter damit beschäftigen. 
Postapokalypse, Idylle, Bukolik, nature writing, Naturlyrik, Dramatik, „Klimawandelroman“, „Ökothriller“, Kinder- und Jugendliteratur, während dieser Aspekt in Frankreich bis jetzt eher vernachlässigt wurde. In Deutschland werden Themen wie das Anthropozän ${ }^{(81)}$, Katastrophen ${ }^{(82)}$, Klimawandel differenziert behandelt, während verwandte Forschungen in Frankreich relativ wenig verbreitet sind. Dies ändert sich langsam, wie es der Call for papers von Justine de Reyniès und Odile Gannier für LOXIAS 52 (März 2016) „(Re)lectures écocritiques: l'histoire littéraire européenne à l'épreuve de la question environnementale“ und der Call for papers von Marc Lacheny und Olivier Hanse (Juni 2018) zum „Catastrophisme environnemental“ für die Revue d'Allemagne belegen.

Der deutsche Ecocriticism ist teilweise auch mit den Cultural Animal Studies verbunden ${ }^{(83)}$, während in Frankreich nur einzelne Ansätze in diese Richtung gehen ${ }^{\left({ }^{(4)}\right.}$. Zur Zeit wird in Deutschland kulturwissenschaftlich zum Thema „Pflanzenkommunikation“(85) geforscht und der neue Forschungsbereich „Cultural Plant Studies“ wird unter anderem von Urte Stobbe im Rahmen der Environmental Humanities vorangetrieben ${ }^{(86)}$. Das Interesse am Nicht-Menschlichen kann sich bis auf das Mineralische erstrecken, wie es eine 2016 im Elsass organisierte deutsch-französische Tagung belegt ${ }^{(87)}$. Ansätze wie Posthumanismus ${ }^{(88)}$, New Materialism, Ökofeminismus und Queer Ecology scheinen im deutschen Ecocriticism mehr verbreitet als in der französischen Ökokritik. Interessant ist auch die medienwissenschaftliche Orientierung des Ecocriticism in Deutschland, die den Akzent auf Intermedialität legt. Medienwissenschaftliche Forschungen umfassen nicht nur literarische Texte, sondern auch nicht-fiktive Texte, Theaterstücke, Performance, Filme, Musik, Comic und andere mediale Ausdrucksweisen ${ }^{(89)}$.

81 Gabriele Dürbeck, Christine Kanz und Ralf Zschachlitz (Hg.), Ökologischer Wandel in der deutschsprachigen Literatur des 20. und 21. Jahrhunderts. Neue Ansätze und Perspektiven, Berlin, Peter Lang, 2018; Caroline Schaumann, Heather Sullivan, German Ecocriticism in the Anthropocene, New York, Palgrave Macmillan, 2017; Gabriele Dürbeck, Jonas Nesselhauf (Hg.), Narrative des Anthropozäns in Kultur und Medien, Frankfurt am Main, Peter Lang, 2018.

82 Gabriele Dürвеск (Hg.), „Klimachaos und Naturkatastrophen in der deutschen Literatur - Desaster und deren Deutung“, in: Franciszek Grucza (Hg.), Vielheit und Einheit der Germanistik weltweit, Frankfurt am Main, Peter Lang (Publikationen der Internationalen Vereinigung für Germanistik, Bd. 14), 2012; Eva Horn, Zukunft als Katastrophe, Frankfurt am Main, Fischer, 2014.

83 Claudia Schmitt und Christiane Solte-Gresser (Hg.), Literatur und Ökologie, Bielefeld, Aisthesis, 2017.

84 Aurélie Choné, „Le message de l'animal dans Indienfahrt de Waldemar Bonsels“, in: Aurélie Choné und Catherine Repussard (Hg.), Des animaux et des hommes/ Von Menschen und Tieren, Recherches germaniques, Sonderheft 10 (2015).

85 Isabel Kranz, Alexander Schwan und Eike Wittrock (Hg.), Floriographie. Die Sprachen der Blumen, Paderborn, Wilhelm Fink, 2016.

86 In November 2017 organisierte sie den Panel „Human Plant Metamorphoses from the Perspective of Cultural Plant Studies“ beim Kongress der Kulturwissenschaftlichen Gesellschaft an der Universität Ghent.

87 Marc Cluet u.a. (Hg.), La dignité du minéral - Cause germanique, cause universelle / Die Würde des Minerals - Ein deutsches und zugleich universelles Anliegen, Würzburg, Königshausen \& Neumann, 2019.

88 Christa Grewe-Volpp und Evi Zemanek (Hg.), „Mensch - Maschine - Materie - Tier. Entwürfe posthumaner Interaktionen“, PhiN. Philologie im Netz: Beihefte, Beiheft 10 (2016).

89 Davon zeugen z.B. die zwei letzten Kapitel des Bandes Literatur und Ökologie (Schmitt/SolteGresser [Anm. 3]) oder der 2018 an der Universität Potsdam (Europäische Medienwissenschaft) in 


\section{Zu einer deutsch-französischen Komparatistik im Feld „Literatur und Ökologie“}

Heutzutage wird der Ecocriticism immer mehr ausdifferenziert. Nach dem ökokritischen Jahrzehnt der 1990er Jahre tauchen neue komplexe Ansätze auf, die interdisziplinäre Praktiken voraussetzen. Wir haben eigentlich nicht mehr einen Ecocriticism, sondern mehrere: Postcolonial Ecocriticism, Material Ecocriticism, Feminist Ecocriticism usw. Und wir haben nicht nur den angelsächsischen Ecocriticism, sondern auch einen deutschen und einen französischen Ecocriticism. Durch ihre rapide internationale Entwicklung stößt nämlich die Ökokritik auf neue Forschungsobjekte und -felder, wie Geopoetik in Frankreich oder Kulturökologie in Deutschland, was zu begrüßen ist, insofern dieser Prozess die Monokultur und Hegemonie des Englischen durch die kulturelle Diversität auf globaler und lokaler Ebene ablöst.

Dieser Beitrag soll jedoch nicht den Eindruck erwecken, dass es eine französische „écocritique“ bzw. einen deutschen Ecocriticism gäbe, die in Konkurrenz zueinander stünden. Die internationale Vernetzung des deutschen Ecocriticism bzw. der französischen „écocritique“ und ihre Ankoppelung an Ansätze aus den Gender Studies (z.B. Ecofeminism), den Postcolonial Studies, dem Poststrukturalismus oder der Diskursanalyse sowie an neue Ansätze wie Ökokosmopolitismus, Biosemiotik oder Multispecies Ethnography ${ }^{(90)}$, und der bedeutende Beitrag französisch- bzw. deutschsprachiger Forscherinnen und Forscher, die nicht in Frankreich (Desblaches, Posthumus, Jaccomard) bzw. Deutschland (Goodbody, Rigby, Wilke) wohnen, wären ausreichende Belege, die man einer solchen essentialistischen Vorstellung entgegenhalten könnte. Claudia Schmitt und Christiane Solte-Gresser bemerken zu Recht:

„Eine europäische Ökokritik, die mit einer Stimme, vielleicht sogar in Abgrenzung zu einer (US)amerikanischen Ökokritik spricht, wird es nicht geben und sollte es auch nicht geben. Wünschenswert wäre allerdings eine aufeinander bezogene Vielstimmigkeit, aus der etwas Neues, Gemeinsames entstehen kann. Denn was sich inzwischen abzeichnet, ist ein deutscher, ein französischer usw. Weg der Ökokritik, welcher in der Tradition der Nationalphilologien und innerhalb der jeweiligen Nationalsprachen verläuft und sich dort zunehmend verzweigt. Damit jedoch wird eine Verknüpfung dieser einzelnen Positionen durch vergleichende und übergreifende Ansätze immer wichtiger “(91).

Die Komparatistin Christiane Solte-Gresser ist der Meinung, dass „angesichts der Sprachen, Nationalgrenzen und Kulturräume überschreitenden Bedeutsamkeit ökokritischer Themen“ eine vergleichende Perspektive ihr besonderes Potential entfaltet ${ }^{(92)}$. Ihr Bemühen um eine europäische Dimension des Ecocriticism, die auch französische Perspektiven miteinbezieht, ist sehr willkommen. Interessant wäre es im Hinblick auf einen komparatistischen deutsch-französischen Ecocriticism, literaturtheoretische Methoden und Methodenreflexionen, Themen (Inhalte, Motive), Formen (Gattungen)

Kooperation mit der Albert-Ludwigs-Universität Freiburg (Medienkulturwissenschaft) organisierte Workshop „Spürtechniken. Von der Wahrnehmung der Natur zur Natur als Medium“.

90 Da finden wir genauso viele Autoren aus Deutschland und Frankreich, z.B. in Frankreich haben Dominique Lestel, Florence Brunois, Florence Gaunet und Vinciane Desprets den Begriff „Etho-Ethnologie“ und „Ethno-Ethologie“ geprägt, um sich von der klassischen anthropologischen Perspektive abzugrenzen und „Zwischenspezifische Beziehungen“ zu situieren, nicht nur menschliche Gemeinschaften.

91 Schmitt/Solte-Gresser, Literatur und Ökologie (Anm. 3), S. 29.

92 Ebd., S. 20. 
und historische Kontexte vergleichend zu betrachten. Inhaltlich ist eine Übereinstimmung bei den behandelten Themen (Katastrophennarrative ${ }^{(93)}$, Pflanzen, Tiere, Klimawandel, Abfall $\left.{ }^{(94)} \ldots\right)$ zu beobachten. Interessant wäre es herauszufinden, weshalb das Thema für den jeweils deutschen bzw. französischen Kontext relevant ist und je nach dem kulturellen Kontext unterschiedlich behandelt wird. Was die Gattungen betrifft, könnten Subgattungen wie Heimatliteratur, Dorfgeschichte, Idylle im deutschsprachigen Raum Genres aus der französischen Literatur gegenübergestellt werden. Die komparatistische Perspektive sollte auch die Wissenstransfers einiger Schlüsselideen zwischen Deutschland und Frankreich kulturgeschichtlich untersuchen, wie zum Beispiel den Begriff „Umwelt“/,milieu“(95), das landschaftliche Denken, den Begriff „Naturdenkmal“/„monument naturel“(96) sowie den kosmologischen Gedanken ${ }^{(97)}$.

In Frankreich wie in Deutschland wurde der Ecocriticism zuerst im Rahmen der Anglistik/Amerikanistik aufgegriffen ${ }^{(98)}$. Ein Interesse an komparatistischen Ansätzen ist auch in französischsprachigen Publikationen der letzten Jahre zu verzeichnen ${ }^{(99)}$. Die Hinwendung zu nicht anglophonen Kulturen nimmt seit einigen Jahren zu; davon zeugen die Arbeiten von Forscherinnen und Forschern aus der Vergleichenden Literaturwissenschaft (Bertrand Guest, Anaïs Boulard), aus der Romanistik (Anne-Laure Bonvalot) und aus der Germanistik (Aurélie Choné). In den USA versuchte schon vor zehn Jahren die Germanistin Sabine Wilke den Ecocriticism aus dem Bereich der Anglistik und Amerikanistik auf andere Fächer wie Hispanistik, Sinologie usw. anzuwenden. Dazu kam der Bereich der Frankoromanistik mit Arbeiten der kanadischen Romanistin Stefanie Posthumus und das Ecolitt-Projekt der Universität Angers. Komparatistische Perspektiven bleiben aber ziemlich selten aufgrund der Dominanz

93 Siehe in Deutschland das von Gabriele Dürbeck geleitete DFG-Forschungsprojekt „Narrative des Anthropozäns in Wissenschaft und Literatur“ (2016-2018) an der Universität Vechta. Siehe in Frankreich z.B. Christian Chelebourg, Les écofictions: mythologies de la fin du monde, Paris, 2012; Anaïs BOULARD, „Un monde à habiter: imaginaire de la crise environnementale dans les fictions de l'Anthropocène“, Dissertation (Vergleichende Literaturwissenschaft), Université d'Angers, 2016.

94 Siehe in Deutschland David-Christopher Assmann, Norbert Otto Eke und Eva Geulen (Hg.), Entsorgungsprobleme: Müll in der Literatur, Berlin, Erich Schmidt Verlag, 2015. Siehe A. BoulARD, „Un monde à habiter" (Anm. 93), in Frankreich.

95 Siehe u.a. Wolf Feuerhahn, „Du milieu à l'Umwelt: enjeux d'un changement terminologique“, Revue philosophique de la France et de l'étranger, 134/4 (2009), S. 419-438.

96 Siehe u.a. Elsa VonAu, „Le paysage et ses lectures: le mouvement de protection de la nature en France et en Allemagne au début du siècle“, in: Guillaume LACQUEMEnT, Karl Martin Born und Béatrice von Hirschinausen (Hg.), Réinventer les campagnes en Allemagne: Paysage, patrimoine et développement rural, Lyon, ENS Éditions, 2013, books.openedition.org/enseditions/4597.

97 Arthur O. Lovejoy, The Great Chain of Being: A Study of the History of an Idea, Harvard, Harvard University Press, 1936. Reprint: New York, Harper \& Row, 2005 (deutsche Übersetzung von Dieter Turck: Die große Kette der Wesen: Geschichte eines Gedankens, Frankfurt am Main, Suhrkamp [taschenbuch wissenschaft], 1993).

98 Seit fünfzehn Jahren hat sich der Ecocriticism in der deutschen und französischen Anglistik und Amerikanistik fest etabliert. Die meisten Forschenden kommen aus der Anglistischen Forschung und der Amerikanistik wie Anne Goarzin, Camille Manfredi, Margot Lauwers, Emmanuelle Peraldo, Clare Sibley-Esposito in Frankreich, Hubert Zapf in Deutschland.

99 A. Suberchicot, Littérature et environnement (Anm. 35); P. Schoentjes, Ce qui a lieu: essai d'écopoétique (Anm. 30). 
der US-amerikanischen Literatur im Feld. Ursula Heise sieht für einen zukünftigen komparatistischen Ecocriticism drei Herausforderungen: „The challenge of non fiction, the challenge of the environmental humanities as a transdisciplinary matrix, and the challenge of the Anthropocene in its tension with posthumanism “(100).

Abschließend möchte ich auf diese Herausforderung, das Potential der Environmental Humanities zu nutzen, näher eingehen. Eine grundsätzliche Fragestellung teilt der Ecocriticism mit den Environmental Humanities, und zwar die Auseinandersetzung mit der Wechselbeziehung zwischen Natur und Kultur und die Infragestellung der traditionellen Natur-Kultur-Dichotomie. Im Bereich des Ecocriticism arbeiten die meisten Forscherinnen und Forscher interdisziplinär mit Geistes- und Naturwissenschaften (Geschichte, Politikwissenschaft, Kunst- und Kulturwissenschaft, Umweltethik, Umweltwissenschaften, Umweltgeschichte, Zoologie, Ethologie usw.). Die gleiche Schwierigkeit bei der Vernetzung der ökokritischen Forschungen betrifft die disziplinübergreifende gemeinsame Forschung in den Environmental Humanities. In Frankreich brachte das von Aurélie Choné, Isabelle Hajek und Philippe Hamman geführte interdisziplinäre Forschungsprojekt der Maison Interuniversitaire des Sciences de l'Homme Alsace (MISHA) „La nature à la lettre. Écritures et production des savoirs sur la nature dans l'Europe moderne et contemporaine“ (2012-2016) Wissenschaftlerinnen und Wissenschaftler aus unterschiedlichen Disziplinen zusammen. 2016 wurde das Ergebnis der Arbeiten im Guide des Humanités environnementales und 2017 im Textbook Rethinking Nature veröffentlicht ${ }^{(101)}$. Diese Sammelbände versuchen die beiden Fallen des Universalismus und Relativismus zu vermeiden, d. h. weder einen universalen, essentialistischen, noch einen relativistischen, konstruktivistischen Natur-Begriff zu vertreten. Sowohl der Ecocriticism als auch die Environmental Humanities wurden aufgrund ihres politisch-ideologischen Corpus kritisiert - denken wir an den Berliner Verlag Matthes \& Seitz, der Botho Strauß' Essay Anschwellender Bocksgesang, Borchardts Anthologie Der Deutsche in der Landschaft (2018), Gerd Bergfleth und Hans-Jürgen Syberberg veröffentlichte ${ }^{(102)}$. Die größte Herausforderung des Ecocriticism und der Environmental Humanities besteht vielleicht darin, nicht der Versuchung einer nostalgischen, romantisierenden, fortschrittskritischen bzw. konservativen Auffassung der Natur zu erliegen.

Die disziplinübergreifende Zusammenarbeit mit Forscherinnen und Forschern aus den Bereichen Geographie, Soziologie, Umweltgeschichte, Literaturwissenschaft usw. wurde 2017-2018 im deutsch-französischen Programm des interdisziplinären Zentrums für Deutschlandstudien und -forschung (CIERA) „Circulations et renouvellement des savoirs sur la nature et l'environnement en France et en Allemagne: vers des humanités environnementales/ Natur und Umwelt: Wissenstransfer und Wissenserneuerung in Frankreich und Deutschland“ weitergeführt. In Deutschland sind

100 Ursula K. Heise, „Comparative Literature and the Environmental Humanities“, 2014, stateofthediscipline.acla.org/entry/comparative-literature-and-environmental-humanities.

101 Choné/Hajek/Hamman, Guide des Humanités environnementales (Anm. 26); Aurélie Choné, Isabelle Hajex und Philippe Hamman (Hg.), Rethinking Nature. Challenging Disciplinary Boundaries, London/New York, Routledge, 2017.

102 Derselbe Verlag hat 2017 (mit dem Bundesamt für Naturschutz) den Deutschen Preis für Nature Writing gestiftet, den erstmals (und zu Recht) Marion Poschmann erhalten hat. 
derartige Projekte ebenfalls vorhanden, wie die 2015 von Gabriele Dürbeck gegründete Naturen-Kulturen-Sektion der Kulturwissenschaftlichen Gesellschaft ${ }^{(103)}$ und die von Jens Soentgen und Hubert Zapf gegründete Augsburger Forschungsgruppe „Environmental Humanities“(104). Solche Initiativen erfordern eine neue Organisation und Verbreitung von Wissen, vor allem mehr Zeit für die reflexive Arbeit (slow scholarship und slow humanity) wie auch eine verstärkte Ankoppelung an das Wirkungsfeld der Politik (sustainibility/Nachhaltigkeit): „Bringing the fruits of such efforts to a wider audience will require environmental humanities scholars to experiment with new ways of organizing and disseminating knowledge“(105). Davon zeugen z.B. neue Initiativen wie das französische Kollektiv ZoneZadir, das anonym und kollektiv Tagungen $^{(106)}$ veranstaltet sowie neue Wege im Umgang mit dem Politischen erforscht. Die Environmental Humanities, die die Literaturwissenschaftlerin Sabine Wilke schon vor 10 Jahren in den USA in Verbindung mit den German Studies gebracht hat ${ }^{(107)}$, können als eine deutliche Erweiterung des Ecocriticism bzw. der écocritique angesehen werden, insofern sie dezidiert interdisziplinär arbeiten und die Naturwissenschaften miteinbeziehen. In der Zukunft könnten sie als Brücke zwischen Ecocriticism und écocritique, d.h. als gemeinsame Grundlage für eine zukünftige komparatistische Ökokritik, dienen.

\section{Zusammenfassung}

Angesichts der verschiedenen kulturellen Traditionen und Umweltdiskursen in Deutschland und Frankreich haben sich die neuen Paradigmen einer umweltbezogenen Literaturwissenschaft im deutschen bzw. französischen Kontext in unterschiedliche Richtungen entwickelt. Im vorliegenden Aufsatz wird erforscht, wie sich der deutsche Ecocriticism bzw. die französische écocritique in Deutschland und Frankreich herausgebildet haben, welche spezifischen Ansätze sie hervorgebracht haben und welche Schnittpunkte/Gemeinsamkeiten sich ergeben. Ohne Anspruch auf Vollständigkeit werden die jeweiligen Entwicklungslinien nachgezeichnet, um Wege aufzuzeigen, wie sich eine deutsch-französische Komparatistik im Feld der Forschungen zum Thema „Literatur und Ökologie" besser entfalten kann.

103 kwgev.wordpress.com/sektionennetzwerke/naturen-kulturen.

104 www.wzu.uni-augsburg.de/Environmental-Humanities-de.html.

105 Hannes Bergthaller, Rob Emmett, Adeline Johns-Putra, Agnes Kneitz, Susanna Lidström, Shane McCorristine, Isabel Pérez Ramos, Dana Phillips, Kate Rigby und Libby Robin, „Mapping Common Ground: Ecocriticism, Environmental History, and the Environmental Humanities“, Environmental Humanities, 5 (2014), S. 261-276, www.environmentalhumanities.org (20.6.2018). Siehe auch Isabelle STENGERs, Une autre science est possible! Manifeste pour un ralentissement des sciences, Paris, Les Empêcheurs de penser en rond, 2013.

106 Wie die Tagung „Habiter les lieux“ im Juli 2018, f-origin.hypotheses.org/wp-content/blogs.dir/4217/ files/2018/06/livret-habiter-les-lieux-v7.pdf.

107 Sabine Wilke (Hg.), „The Environmental Humanities“, Special Issue, Pacific Coast Philology (2011); Sabine Wilke, Japhet Johnstone (Hg.), Readings in the Anthropocene: The Environmental Humanities, German Studies, and Beyond, London, Bloomsbury, 2011. 


\begin{abstract}
Résumé
Compte tenu des différences de traditions culturelles et de discours environnementaux en France et en Allemagne, les études littéraires axées sur les questions environnementales ont déployé leurs nouveaux paradigmes dans des directions différentes en contexte français et allemand. La présente contribution analyse les chemins particuliers suivis par l'Ecocriticism allemand et l'écocritique française, les champs de recherche spécifiques qu'ils ont privilégiés et les points de convergence/comparaison qui peuvent émerger entre eux. Sans viser à l'exhaustivité, cet article brosse les grandes lignes de déploiement dans chaque contexte, afin de mettre en évidence des pistes pour une perspective comparative franco-allemande dans le domaine des relations entre littérature et écologie.
\end{abstract}

